\title{
1
}

\section{Adaptive Structures for Structural Health Monitoring}

\section{Daniel J. Inman and Benjamin L. Grisso}

Center for Intelligent Material Systems and Structures, Department of Mechanical Engineering, 310 Durham Hall, Mail Code 0261, Virginia Tech, Blacksburg, VA24061, USA

\subsection{INTRODUCTION}

For some time the adaptive structures community has focused on transducer effects, and the closest advance into actually having a structural system show signs of intelligence is to include adaptive control implemented with a smart material. Here we examine taking this a step further by combining embedded computing with a smart structural system in an attempt to form an autonomous sensor system. The focus here is based on an integrated structural health monitoring system that consists of a completely wireless, active sensor with embedded electronics, power and computing. Structural health monitoring is receiving increased attention in industrial sectors and in government regulatory agencies as a method of reducing maintenance costs and preventing disasters. Here we propose and discuss an integrated autonomous sensor 'patch' that contains the following key elements: sensing, energy harvesting from ambient vibration and temperature, energy storage, local computing/decision making, memory, actuation and 
wireless transmission. These elements should be autonomous, self-contained and unobtrusive compared to the system being monitored. Each of these elements is discussed as a part of an integrated system to be used in structural health monitoring applications.

In addition, the concept of using smart materials in a combined monitoring and self-healing function is briefly discussed. This chapter concludes with some thoughts on the way forward in monitoring which is a subset of the newly formed area called 'autonomic structures' and includes a short introduction to such systems.

Autonomous sensing requires the integration of a number of subsystems: power, sensor material, actuation material, energy management, telemetry and computing. This chapter discusses one such solution to building an autonomous sensing system as well as steps taken to further integrate such a system into a load-bearing adaptive structure. The basic idea of the autonomous sensing system proposed here is summarized in Figure 1.1.

The proposed sensing system must have the following components in order to function autonomously. First, it must be built around a transducer material that performs the basic sensing function. For the example discussed here, this material consists of a piezoceramic, which produces an electric field when strained (see, for instance, Dosch et al., 1994). The electric field is then converted to a voltage, which is proportional to local strain and can be used to measure local displacement or velocity. Here, however, we are interested in measuring the electrical impedance of the sensing piezoceramic (PZT in this case) as discussed below. Figure 1.1 also indicates that the PZT serves as an actuator as well. Actuation is needed because many of

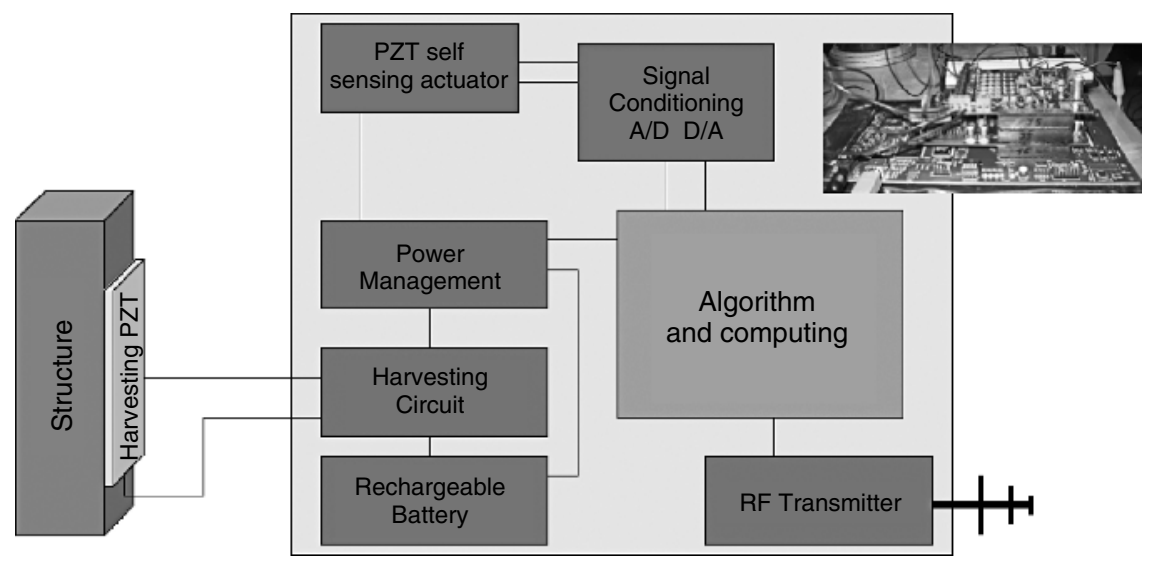

Figure 1.1 A proposed autonomous sensing system 
the best algorithms for structural health monitoring (SHM) require a known input (Doebling et al., 1998) in order to form measurements resembling a transfer function. At the very least, input-output measurements contain much more information than output only measurements. The existence of this actuation element separates the proposed active sensing system from many of the wireless sensing systems proposed by others (such as the Mote system). The circuit of Dosch et al. (1994) allows 'self-sensing actuation' and results in a reduction in the number of required components, reducing the size and weight requirements.

The second key element in the proposed autonomous sensing scheme is the use of a local computing platform. In a review of smart sensing technology for civil applications, smart sensors are defined as sensors which contain an onboard microprocessor giving the system intelligence capabilities (Spencer et al., 2004). Several sensor platforms have incorporated microprocessors for the purpose of power management and signal conditioning using off-the-shelf chips. The system here takes the approach that (a) it takes less energy to compute than to transmit raw data, and (b) at some point during the sensor's life, it may be desirable to remotely change the algorithm used to determine damage. This is also the area in which further autonomy can be gained by enabling the sensor to make decisions. The philosophy of this approach is to make all the calculations at the sensor location and to broadcast only a limited amount of information in the form of a decision. Localized computing and decision again separates the proposed system from many of the previous efforts in the literature (Straser and Kiremidjian, 1998; Giurgiutiu and Zagrai, 2002; Lynch et al., 2002, 2003). However, Lynch et al. (2004a, b) also use atthe-sensor computing to perform a time series analysis and broadcasts results, rather than raw data streams. A Berkeley-Mote platform is also used as a basis for a wireless structural health monitoring system with an embedded damage detection algorithm (Tanner et al., 2003). A main difference between the approach presented here and other approaches is that they use a standard operating system whereas the goal here is to diminish the operating system to further reduce the power required to run the system.

The third key element of the system of Figure 1.1 is the power harvesting, management and storage system. Most systems to date use batteries as the source, and our goal here is to extend the autonomy of the sensor system by using various energy harvesting methods, power management and energy storage devices. The transmission device is taken as a standard off-the-shelf system here (see Lynch and Koh, 2005), and no new results are offered in the telemetry area. The main thrust of the work proposed here is to examine energy conservation through using a digital signal processor (DSP) platform without using an operating system (which tends to waste energy). 


\subsection{STRUCTURAL HEALTH MONITORING}

Damage prognosis (DP) is the prediction in near real time of the remaining useful life of an engineered system given the measurement and assessment of its current damaged (or aged) state and accompanying predicted performance in anticipated future loading environments (Inman et al., 2005). Self-healing can be thought of as structural repair of damage. A key element in damage prognosis and self-healing is obviously that of structural health monitoring (SHM). The added effort in damage prognosis is the concept of organizing the ability to make a decision based on the current assessment of damage by assuming future loads and predicting how the damaged system will behave. This prediction is then used to make a decision about how to use the damaged structure (or if to use it) going forward. A military aircraft hit by enemy fire gives a simple example of a prognosis system. The ideal prognosis system would detect the damage and inform the pilot if he/she should bail out, ignore the damage or perhaps continue to fly by under reduced flight performance. The battery indicator on a laptop performs a similar prediction in the sense that it measures current usage and estimates the remaining time left before required shutdown.

The added effort in self-healing is repairing the damage to return the structure to a usable state. A simple example is given below of a selfhealing mechanism, while 'Self-healing composite materials' in dealt with in Chapter 9 of this volume. In the example given below of a self-healing bolted joint, there is a need to know the extent of the damage before self-repair can begin. Again, the concept of determining the state of the structure's health and the extent of its damage is a key element in the process. In this sense, damage prognosis and damage mitigation are natural extensions to SHM and can be viewed as the next steps.

In order of increasing difficulty, damage monitoring and prognosis problems can be categorized in the following stages of increasing difficulty:

1. Determining the existence of damage.

2. Determining the existence and location of damage.

3. Determining the existence, location and characterization (quantification) of damage.

4. All of the above and predicting the future behavior under various loads (damage prognosis).

5. All of the above and mitigating the effects of damage (self-healing structures). 
6. Combining problems $1,2,3$ or 4 with smart materials to form selfdiagnosing Structures.

7. Combining the above with adaptive structures to form autonomous, selfrepairing structures (autonomic structures).

Adaptive materials, or smart materials, and structures integrate very nicely into all seven of these problems. In the following, several examples are given to illustrate the effect that integrating these two disciplines has on solving problems arising in damage prognosis and mitigation, with the goal of eventually producing an entirely standalone chip fully integrated into a structure.

There are numerous SHM algorithms. A review of the SHM literature (Doebling et al., 1998; Sohn et al., 2003; Inman et al., 2005) indicates that the main drawbacks and issues of the current SHM methods include:

1. Spatial aliasing: Conventional monitoring is accomplished with a limited number of sensors dispersed over a relatively large area of a structure providing poor spatial resolution and thus is only capable of detecting fairly significant damage.

2. Cabling issues: As a new generation of sensing technologies and sensor arrays pushes the limits of scale, the cabling and bookkeeping of sensor arrays has become an issue. Although wireless communication technology can provide a partial solution to this problem, unwavering power supply to the transmitter remains largely unsolved.

3. Environmental issues: Varying environmental and operational conditions produce changes in the system's dynamic response that can be easily mistaken for damage.

4. Integration issues: The predominant approach is to design separate systems leading to inefficiencies and reduced capabilities that could be increased through an integrated design philosophy.

Much activity has emerged in the area of wireless sensing (see, for instance, Lynch et al., 2003). However, few have focused on the power requirements or on the integration of the algorithms into the choice of sensing hardware. In summary, the basic roadblock in adapting SHM methods in practice is that commercial sensing systems have not been developed with the intent of specifically addressing these drawbacks. The need to develop a system that goes beyond the laboratory demonstration and can be deployed in the field on real-world structures necessitates the goal of this effort: that new sensing 
hardware must be developed in conjunction with software interrogation algorithms.

The SHM algorithm used here is called the impedance method, was introduced by Liang et al. (1994), was used extensively over the last 10 years and is described next. Other algorithms, such as Lamb wave methods or vibration-based methods, can also be used, but, for the sake of simplicity and example, only impedance methods are discussed here.

\subsection{IMPEDANCE-BASED HEALTH MONITORING}

Impedance-based health monitoring techniques utilize small piezoceramic (PZT) patches attached to a structure as self-sensing actuators to simultaneously excite the structure with high-frequency excitations and monitor changes in the patch electrical impedance signature (Park et al., 2003). Since the PZT is bonded directly to the structure of interest, it has been shown that the mechanical impedance of the structure is directly correlated with the electrical impedance of the PZT (Liang et al., 1994). Thus, by observing the electrical impedance of the PZT, assessments can be made about the integrity of the mechanical structure.

The impedance-based health monitoring method is made possible through the use of piezoelectric patches bonded to the structure that act as both sensors and actuators on the system. When a piezoelectric is stressed, it produces an electric charge. Conversely, when an electric field is applied, the piezoelectric produces a mechanical strain. The patch is driven by a sinusoidal voltage sweep. Since the patch is bonded to the structure, the structure is deformed along with it and produces a local dynamic response to the vibration. The area one patch can excite depends on the structure and material. The response of the system is transferred back from the piezoelectric patch as an electrical response. The electrical response is then analyzed and, since the presence of damage causes the response of the system to change, damage is shown as a phase shift and/or magnitude change in the impedance.

The solution to the wave equation gives the following equation for electrical admittance as a function of the excitation frequency $\omega$ :

$$
Y(\omega)=i \omega a\left(\bar{\varepsilon}_{33}^{T}(1-i \delta)-\frac{Z_{s}(\omega)}{Z_{s}(\omega)+Z_{a}(\omega)} d_{3 x}^{2} \hat{Y}_{x x}^{E}\right)
$$

In Equation (1.1), $Y$ is the electrical admittance (inverse of impedance), $Z_{a}$ and $Z_{s}$ are the PZT material's and the structure's mechanical impedances, respectively, $Y_{x x}^{T}$ is the complex Young's modulus of the PZT with zero electric field, $d_{3 x}$ is the piezoelectric coupling constant in the arbitrary $x$ 
direction at zero stress, $\varepsilon_{33}^{T}$ is the dielectric constant at zero stress, $\delta$ is the dielectric loss tangent of the PZT, and $a$ is a geometric constant of the PZT. This equation indicates that the electrical impedance of the PZT bonded onto the structure is directly related to the mechanical impedance of a host structure.

The impedance method has many advantages compared to global vibrationbased and other damage detection methods. Low excitation forces, combined with high frequencies (typically greater than $30 \mathrm{kHz}$ ), produce power requirements in the range of microwatts. The small wavelengths at high frequencies also allow the impedance method to detect minor local changes in structural integrity and, in some cases, imminent damage.

The impedance method has been used successfully to warn of impending damage in a number of different experiments and field tests. These range from simple laboratory tests to illustrating the method on the NASA Space Shuttle launch tower.

Traditionally, the impedance method requires the use of an impedance analyzer. Such analyzers are bulky and expensive, and are not suited for permanent placement on a structure. With the current trend of SHM heading towards unobtrusive self-contained sensors, the first steps in meeting the low-power requirements resulted in the MEMS-Augmented Structural Sensor (MASSpatch) (Grisso et al., 2005).

The use of a relatively small resistive circuit instead of the impedance analyzer was made possible by Peairs et al. (2004a). The idea of the 'low-cost' impedance-measuring device is to remove the need for a bulky analyzer and replace it with an operational-amplifier-based device. Impedance measurements can then be generated utilizing an FFT analyzer and small current measuring circuit. FFT analyzers, such as those used in modal analysis, are much more common and less expensive than impedance analyzers and are available on a chip.

In fact, Analog Devices has also recently introduced impedance measurement devices in chip format. The AD5933 has a 1 MSPS sampling rate and also comes in an evaluation board format. A prototype similar to MASSpatch has been developed using the AD5933 evaluation board, an ATmega128L microprocessor, and Xbee radios for wireless communications (Mascarenas et al., 2006). Using the microprocessor to control the evaluation board, bolt loosening was detected in a frame structure.

In contrast, the system described here is based on a single board computer system, which interrogates a structure utilizing a self-sensing actuator and the low-cost impedance method. All the structural interrogation and data analysis are performed in near real time at the sensor location. Wireless transmissions alert the end user to any harmful changes in the structure. The first version 
of this (MASSpatch) had some limitations. The algorithm, written in C, to perform the impedance method was utilized as an executable in the DOS operating system. When using an operating system, much of the processing power is used to run the actual system, as well as the algorithm. Determining how much energy is used for calculating the actual algorithm is difficult. Also, a digital-to-analog converter (DAC) was never fully incorporated into the system and reliance on an external function generator was needed for structural excitation. For these reasons, a new processing device must be used in order to optimize the prototype. The current system is based on a digital signal processor (DSP) platform. The benefits of this new system proposed here are discussed, along with current research and the path forward to a complete standalone SHM system.

\subsection{LOCAL COMPUTING}

To implement the impedance-based SHM method in a field-deployable setup, hardware is assembled as shown in Figure 1.2. Using the low-cost technique, accurate approximations of the structural impedance can be determined without complex and expensive external electronic analyzers. As shown in Figures 1.2 and 1.3, all of the hardware needed to utilize the impedance method is condensed into a single stacked board configuration. A description of each of the components follows.

This prototype is based on a TMS320C6713 DSK evaluation DSP module from Texas Instruments (Texas Instruments, 2005b). The DSP has an internal system clock speed of $225 \mathrm{MHz}, 192 \mathrm{kB}$ of internal memory, and external synchronous dynamic random access memory (SDRAM) of $16 \mathrm{MB}$. With a large amount of external memory, the memory space is partitioned into two major sections: samples for DAC output, and samples from the analogto-digital converter (ADC). As shown in Figure 1.2, the ADC, DAC and

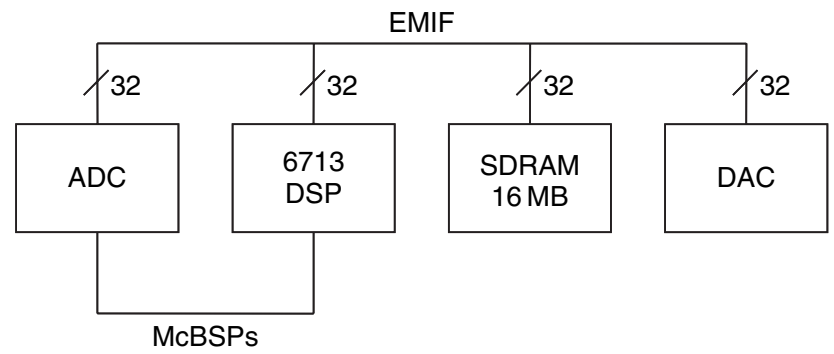

Figure 1.2 A diagram of the proposed hardware configuration 
SDRAM all share an external memory interface (EMIF). The DSP controls the ADC by means of a multichannel buffered serial port (McBSP) acting in general purpose input-output (GPIO) mode.

Two more evaluation boards from Texas Instruments are used as the ADC and DAC. The ADS8364 EVM ADC board has six channels of input and a $250 \mathrm{kHz}$ sampling rate (Texas Instruments, 2002). Conversion resolution for the ADC board is 16 bits. For the DAC, a TLV5619-5639 EVM board is used with a 5639 DAC (Texas Instruments, 2001). The DAC evaluation board has two outputs and a maximum sampling rate of $1 \mathrm{MHz}$ at 12 bit resolution. The physical orientation of the DSP kit, ADC and DAC can be seen in Figure 1.3 (see also Table 1.1).

The wireless transmitter and receiver are used to indicate the current state of damage for the structure of interest. The transmitter sends a quantified amount of damage, and the receiver displays this value on a host computer. The current prototype uses Radiometrix RX2M-458-5 and TX2M458-5 wireless sensors as the receiver and transmitter (Radiometrix Ltd., 2005).

The operational flow of the current prototype allows SHM to be performed all with one piece of hardware. The DSP board controls the entire operation. An excitation signal is sent from the DAC board simultaneously to the ADC board and the structure of interest. The ADC reads the voltage signal from the DAC and simultaneously reads the voltage across the sensing resistor

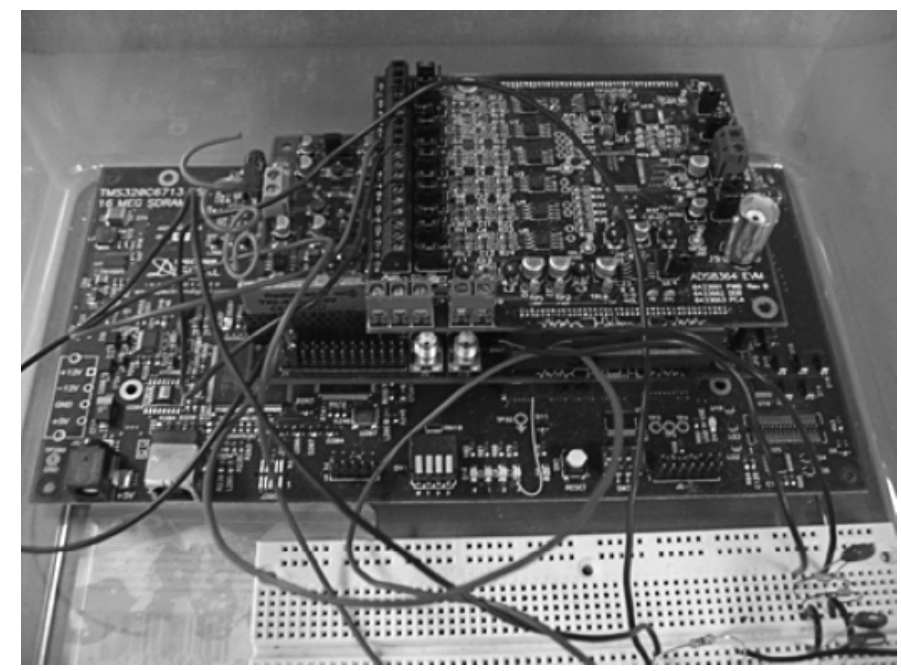

Figure 1.3 The prototype is shown with the DSP on the bottom followed by the $\mathrm{DAC}$ and $\mathrm{ADC}$ 
Table 1.1 Specifications for the prototype of Figure 1.3

\begin{tabular}{|c|c|c|c|c|c|}
\hline \multicolumn{2}{|c|}{ Processing/programming } & \multicolumn{2}{|c|}{ Sensing/sampling } & \multicolumn{2}{|c|}{ Wireless transmission } \\
\hline Processor & $\begin{array}{l}\text { TMS320C6713 } \\
225 \mathrm{MHz} \text { floating } \\
\text { point DSP }\end{array}$ & $\begin{array}{l}\mathrm{ADC} \\
\text { resolution }\end{array}$ & 16 bit & $\begin{array}{l}\text { Usable } \\
\text { range }\end{array}$ & Over $1 \mathrm{~km}$ \\
\hline $\begin{array}{l}\text { Internal } \\
\text { memory }\end{array}$ & $192 \mathrm{~KB}$ & $\begin{array}{l}\text { Max } \\
\text { sampling } \\
\text { frequency }\end{array}$ & $\begin{array}{l}250 \mathrm{kHz} \text { per } \\
\text { channel } \\
750 \mathrm{kHz} \\
\text { paired }\end{array}$ & $\begin{array}{l}\text { Operating } \\
\text { frequencies }\end{array}$ & $\begin{array}{l}433.05- \\
434.79 \mathrm{MHz}\end{array}$ \\
\hline $\begin{array}{l}\text { External } \\
\text { memory }\end{array}$ & $16 \mathrm{MB}$ SDRAM & $\begin{array}{l}\text { Sensor } \\
\text { types and } \\
\text { ranges }\end{array}$ & $\begin{array}{l}6 \text { analog at } \\
\text { up to }+10 \\
\text { to }-10 \mathrm{~V}\end{array}$ & $\begin{array}{l}\text { Data bit } \\
\text { rate }\end{array}$ & $5 \mathrm{kbps}$ \\
\hline \multicolumn{3}{|l|}{ Actuation } & \multicolumn{3}{|c|}{ Dimensions } \\
\hline \multirow{3}{*}{\multicolumn{2}{|c|}{$\begin{array}{l}\text { DAC resolution } \\
\text { Max sampling frequency } \\
\text { Channels }\end{array}$}} & 12 bit & \multirow{3}{*}{\multicolumn{2}{|c|}{$\begin{array}{l}\text { DSP board } \\
\text { DAC board } \\
\text { ADC board } \\
\text { Height }\end{array}$}} & $22 \times 11.5 \mathrm{~cm}$ \\
\hline & & $1 \mathrm{MHz}$ & & & $13.5 \times 8.5 \mathrm{~cm}$ \\
\hline & & 2 up to $4 \mathrm{~V}$ & & & $\begin{array}{l}10 \times 8.5 \mathrm{~cm} \\
4.5 \mathrm{~cm}\end{array}$ \\
\hline
\end{tabular}

(seen in the foreground of Figure 1.3) of the low-cost impedance circuit. After 10 excitation cycles, the signals are averaged, a FFT is performed, and one impedance measurement is generated. The first two measurements generated are baseline impedance curves. Once the baseline is stored, each measurement is compared to the baseline to determine by means of a damage metric whether there is damage in the structure.

Impedance signatures are, in general terms, simply frequency response functions (FRFs). They have the general appearance of FRFs, as seen in Figure 1.6 below. By monitoring the changes in the peaks of these FRFs, a simple damage algorithm can be used to quantify the amount of change in the peaks and thus the amount of damage in the structure. In this case, a variation of the root mean square deviation is used as the damage metric (Park et al., 2003).

In order to excite the structure of interest, a sine cardinal, or simply sinc, was used as the DAC output. The sinc function has the unique property in that its Fourier transform is a box. Having a uniform value in the frequency domain allows for a band of frequency content in one pulse. The sinc function is based on a fundamental frequency and then frequencies which build upon the fundamental, as shown in Figure 1.4. By slightly altering the fundamental 


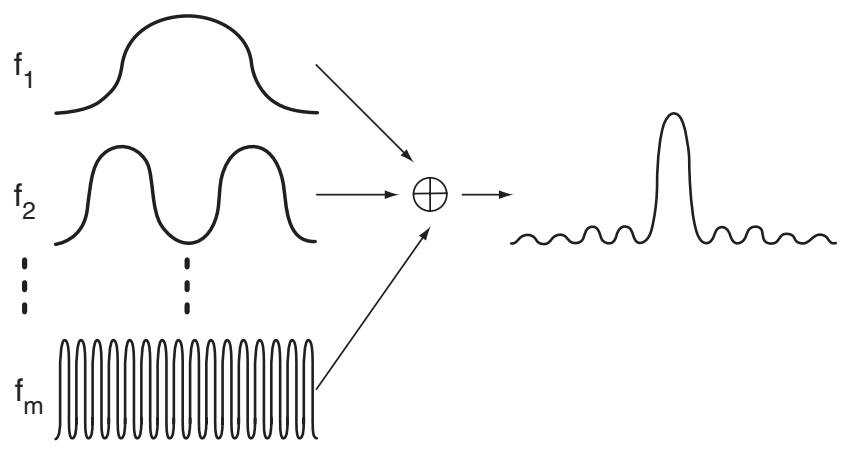

Figure 1.4 A diagram showing the sinc function (on the right) and how the function is built

frequency each time a pulse is sent out, the averaged spectrum is even smoother. The sinc function is described as (Schilling and Harris 2005)

$$
\operatorname{sinc}=\frac{\sin (x)}{x}
$$

By using a sinc function instead of exciting the structure with discrete frequencies, more frequencies can be excited in the same amount of time. The auto spectrum of the output signal will also be a straight line over all the frequencies excited. Other advantages of the sinc function include needing less memory space and less traffic in the external interface, as well as lower power consumption in the DSP, ADC and DAC, a key factor for self-powered systems.

\subsection{POWER ANALYSIS}

Currently, the prototype runs off of DC power supplies. In a permanent setting, the prototype will operate off of battery power recharged by harvested ambient energy. To optimize the battery life and minimize the required maintenance schedule, both piezoelectric and thermal-based power harvesting can be utilized to recharge batteries (see below). Piezoelectric materials have the unique property of being able to transform mechanical strain into an electric charge. By using this property, piezoelectrics can harvest energy by using a system's own ambient motion, transform this mechanical kinetic energy into electrical potential, and store the electrical energy, power devices, or recharge a battery using power harvesting circuitry (Sodano et al., 2003). 
In order to prepare the prototype to be completely run off of a battery and power harvesting, a complete power analysis is done of the current system. In the prototype, the DSP board supplies power to the DAC board, which in turn supplies power to the wireless transmitter. Due to the connectivity of these systems, the power consumption of the DAC and transmitter cannot be exactly determined. However, they can be estimated. According to specifications, the maximum current the transmitter consumes is $100 \mathrm{~mA}$ at $5 \mathrm{~V}_{\mathrm{DC}}$, or $0.05 \mathrm{~W}$ (Radiometrix Ltd., 2005). The transmitter is supplied with $3.3 \mathrm{~V}_{\mathrm{DC}}$, and only sends out a very small signal, so $0.33 \mathrm{~W}\left(100 \mathrm{~mA}\right.$ at $\left.3.3 \mathrm{~V}_{\mathrm{DC}}\right)$ is a high estimate. The DAC board is stated to consume $170 \mathrm{~mA}$ at $3.3 \mathrm{~V}_{\mathrm{DC}}$ and $150 \mathrm{~mA}$ at $5 \mathrm{~V}_{\mathrm{DC}}$, or a range of 0.561 to $0.825 \mathrm{~W}$ (Texas Instruments, 2001). In this setup, the DAC board is being operated at $5 \mathrm{~V}_{\mathrm{DC}}$.

The DSP board is supplied with a $5 \mathrm{~V}_{\mathrm{DC}}$ power supply, so the DSP, DAC and wireless transmitter can be measured as a group. When the system is fully turned on, but the algorithm is not being performed, the DSP requires $470 \mathrm{~mA}$ at $5 \mathrm{~V}_{\mathrm{DC}}$, which is $2.35 \mathrm{~W}$. While the whole impedance-based SHM operation is being performed, including wireless transmission, the current draw increases to $570 \mathrm{~mA}$, giving a power of $2.85 \mathrm{~W}$. So, wireless transmissions are shown not to be a significant drain on the power supply. All of these measurements are instantaneous power, but the current draw remained almost constant during a complete operational cycle.

The ADC has its own $\pm 12 \mathrm{~V}_{\text {DC }}$ power supply. During operation, the ADC requires $60 \mathrm{~mA}$, yielding $1.44 \mathrm{~W}$ of power. So, the total amount of power required for the prototype to completely perform impedance-based SHM is $4.29 \mathrm{~W}$. A summary of the power analysis can be seen in Table 1.2. Comparatively, the MASSpatch prototype used around $4.5 \mathrm{~W}$ of power (Grisso et al., 2005); $4.29 \mathrm{~W}$ does not seem like a significant reduction considering the advances in hardware and excitation efficiency, but the previous (MASSpatch) prototype relied on an external function generator to provide excitations. MASSpatch did not include its own DAC, and the function generator used was plugged into a wall outlet and consumes a considerable amount of power.

Table 1.2 The power consumption for the prototype components

\begin{tabular}{ll} 
Power analysis & \\
\hline Wireless (estimate) & $0.33 \mathrm{~W}(\max )$ \\
DAC (estimate) & $0.825 \mathrm{~W}(\max )$ \\
DSP, DAC, wireless group & $2.85 \mathrm{~W}$ during operation \\
ADC & $1.44 \mathrm{~W}$ \\
TOTAL & $4.29 \mathrm{~W}$ \\
\hline
\end{tabular}


Even with $4.29 \mathrm{~W}$ of power, the prototype is capable of being run solely off of battery power and piezoelectric power harvesting. For instance, if the prototype was being continuously run for 10 minutes or more (a rather excessive time), $101.2 \mathrm{~V}, 200 \mathrm{mAh}$ capacity batteries could supply more than enough energy to the system. A 1 Ah capacity means that a battery will last for $1 \mathrm{~h}$ if it is subjected to a discharge current of $1 \mathrm{~A}$. A $200 \mathrm{mAh}$ battery can be recharged to $90 \%$ capacity in $1.2 \mathrm{~h}$ with a random vibration signal at 0 to $500 \mathrm{~Hz}$ if a $6.35 \times 2.375$ inch PZT is used (Sodano et al., 2003).

The prospect for success of the proposed autonomous sensor can be captured in some simple energy accounting. We require

$$
E_{H}(t)+E_{H}(0)>E_{C}(t)
$$

in which $E_{H}(t)$ is the total energy harvested until time $t$ from the beginning of a sensor's cycle, $E_{H}(0)$ is the initial energy stored in storage elements at the beginning of the cycle, and $E_{C}(t)$ is the total energy consumed during the period for data collection, computation, transmission. If the above equation cannot be met at a certain time instant $t$, then the duty cycle, environment and monitoring task will not work with the proposed autonomous sensor.

\subsection{EXPERIMENTAL VALIDATION}

To validate our prototype concept, the system's capabilities are demonstrated in the laboratory. A bolted joint, as seen in Figure 1.5, is tested for the initial experiments. The bolted joint structure consists of two aluminum beams connected with four bolts. A piezoelectric patch is attached to this structure; the piezoelectric acts as a self-sensing actuator. Damage is induced in the bolted joint by tightening or loosing one or more of the bolts.

Using traditional impedance techniques (a HP 4194A impedance analyzer), a standard for the bolted joint experiment is generated for comparison to results from the prototype. Initial bolted joint testing shows that the impedance method readily detects damage induced by loose bolts. Slightly

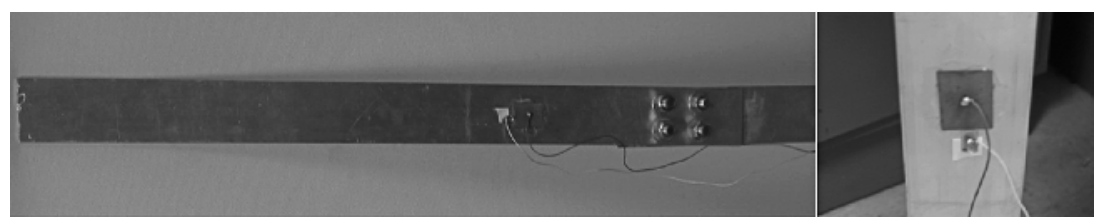

Figure 1.5 Images of the bolted joint and piezoelectric patch are shown 


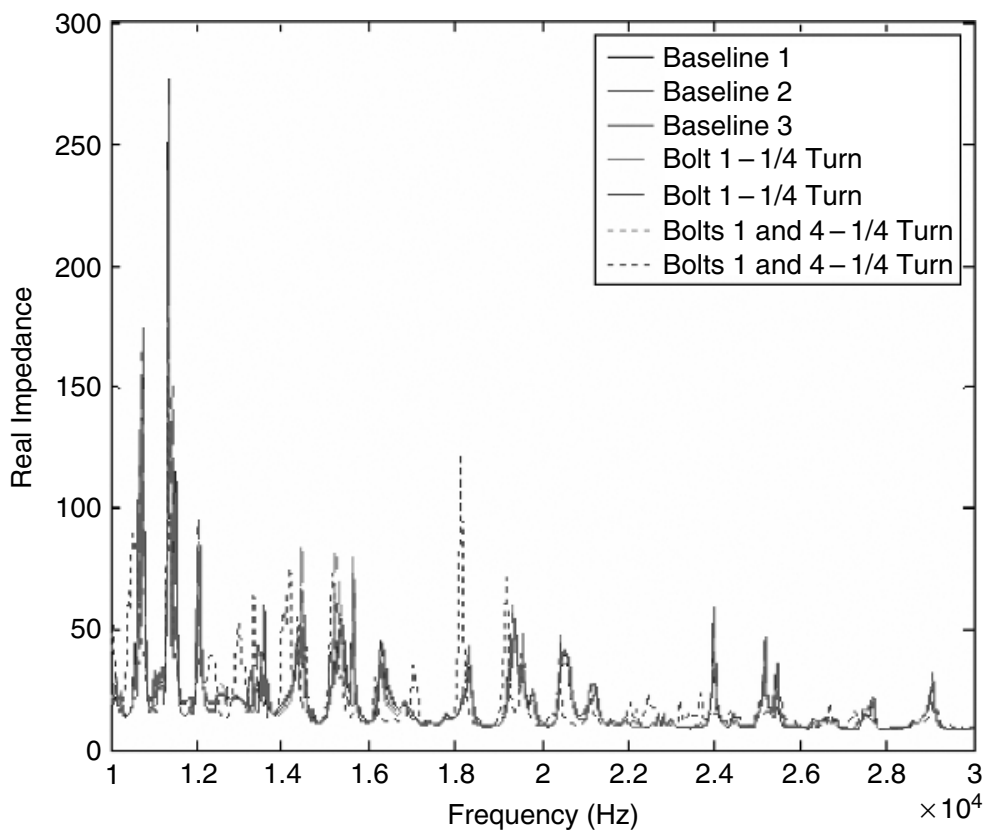

Figure 1.6 The baseline of the real value of impedance vs. frequency and damaged impedance signatures for the bolted joint are shown

loosening only one of four bolts significantly changes the impedance signature. Figure 1.6 shows the impedance curves generated using an impedance analyzer.

As displayed in Figure 1.6, the peaks of the impedance signature change as damage is introduced to the structure by loosening bolts. The more the structure is damaged, the more the peaks shift from the baseline. A frequency range of $10-30 \mathrm{kHz}$ was selected for easy comparison to the prototype. A variation root mean square deviation (RMSD) damage metric is utilized to analyze changes in these peaks and determine the amount of damage present. Figure 1.7 displays this damage metric in bar chart form.

In Figure 1.7, the first two bars compare the second and third baselines (healthy measurements) to the first baseline. The next two groups of bars compare the next two damage cases, the loosening of bolt one and the combination of bolts 1 and 4 .

Using the same bolted joint, the prototype could be directly compared to standard impedance measurement methods. Code Composer Studio software allows for visualization of what the damage detection algorithm is doing in the DSP core (Texas Instruments, 2005a). At each step in the algorithm, the real 


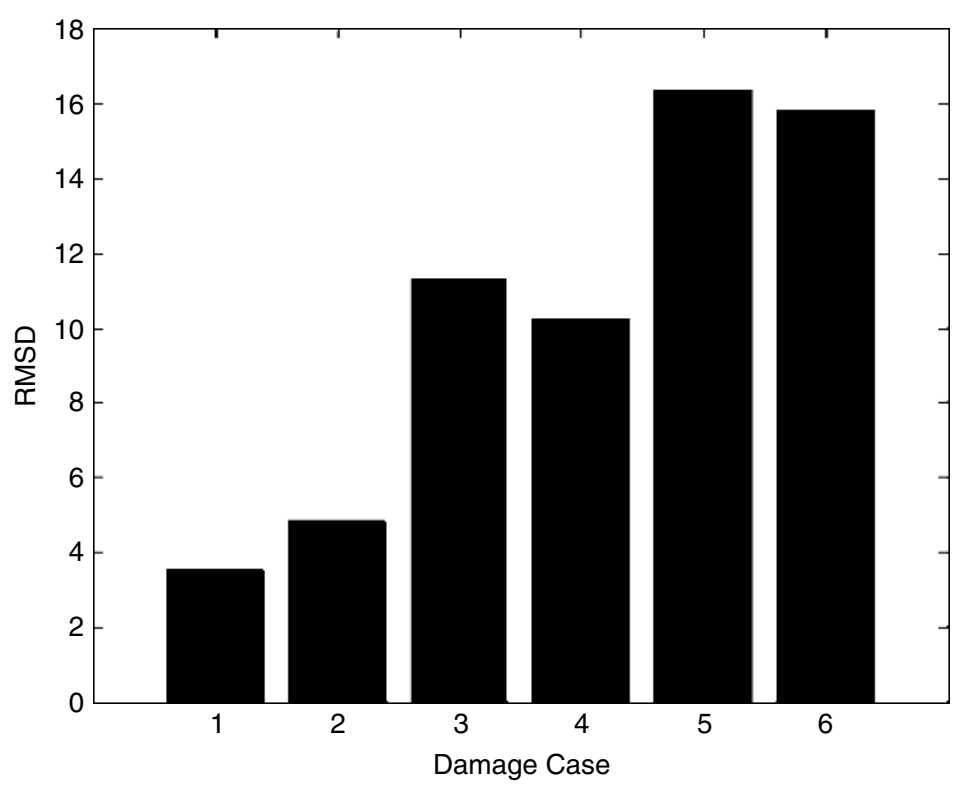

Figure 1.7 The damage metric compares the baselines and damaged curves

impedance measurement (as data is acquired) is displayed along with the baseline, the averaged real impedance measurement used to compare to the baseline, the original DAC sinc function output, and the ADC sampled output. The most important part of the display is the damage metric value, which is updated with each measurement to indicate how much damage is present in the structure. Impedance measurements are taken over the range of $10-30 \mathrm{kHz}$.

The spectrum of the output can also be displayed, as shown in Figure 1.8. As expected from a sinc function, the auto spectrum is a flat line indicating that every frequency of interest is being excited. In Figure 1.8, it should be noted that 512 frequency components are displayed, representing 0 to $64200 \mathrm{~Hz}$. In reality, only half of the spectrum is used, so 256 frequency lines represent 0 to $32100 \mathrm{~Hz}$.

Initially, measurements are taken with all of the bolts completely tightened. With no damage to the structure, the baseline and damaged impedance signature should be the same. As Figure 1.9 shows, the impedance curves for the new measurement and original baseline are almost identical. Figure 1.9 is generated by Code Composer Studio, and allows for graphical displays of what is actually occurring at specific memory locations in the hardware. All of the computations are performed on the DSP, and the graphs just show the results. The damage metric value displayed is 0.02 . 


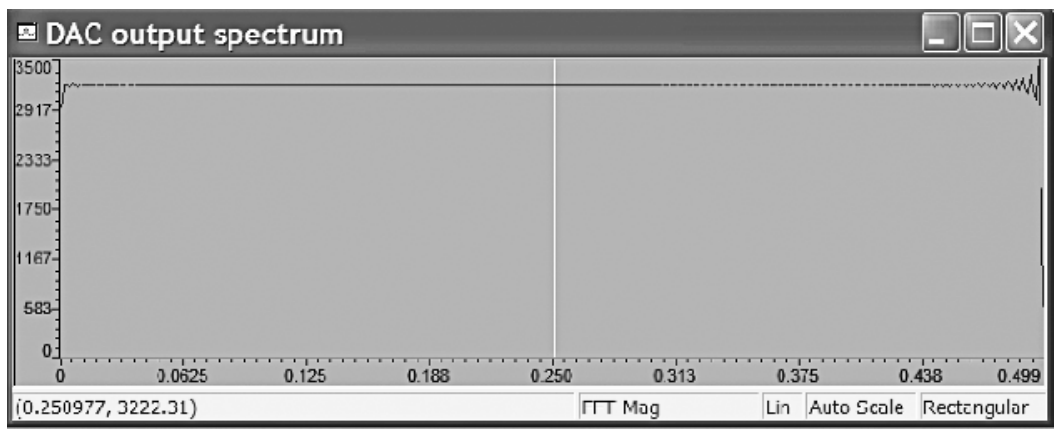

Figure 1.8 The auto spectrum is displayed for the sinc function

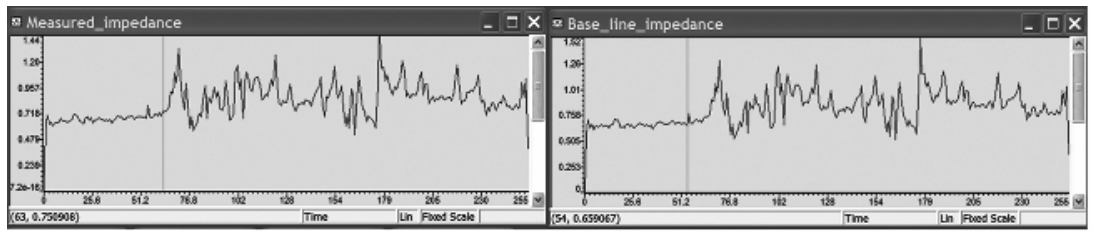

Figure 1.9 The measurement with no damage to the structure is compared to the baseline

One interesting thing to note is that the impedance signatures from Figure 1.9 and Figure 1.6 are very similar. Both show a good number of peaks in similar locations over the range of $10-30 \mathrm{kHz}$. Also, the frequency is displayed by a frequency index, $i$, from 0 to 256 , where $i=256$ corresponds to $32100 \mathrm{~Hz}$. The RMSD is then taken over the frequency indices of $i=79$ to 256 (or $9906-32100 \mathrm{~Hz}$ ), even though the whole frequency range from 0 to $32100 \mathrm{~Hz}$ is displayed. Now, a small amount of damage was induced on the bolted joint by loosening one of the bolts a quarter turn. With just this small amount of damage, the prototype easily recognizes the difference as shown in the peak changes of the measured impedance seen in Figure 1.10.

Comparing the two curves in Figure 1.11, the damage metric increased to 0.13 . This is a $550 \%$ change from the original damage metric. With only a little bit of damage, the damage metric easily indicates that the structure has changed. Next, a second bolt was also slightly loosened. Figure 1.11 displays the difference for this damage case.

As seen in Figure 1.11, with even more damage, the peaks of the measured impedance signature change even more. The damage metric also notices the change and calculates a new value of 0.21 . Utilizing a bolted joint, the 


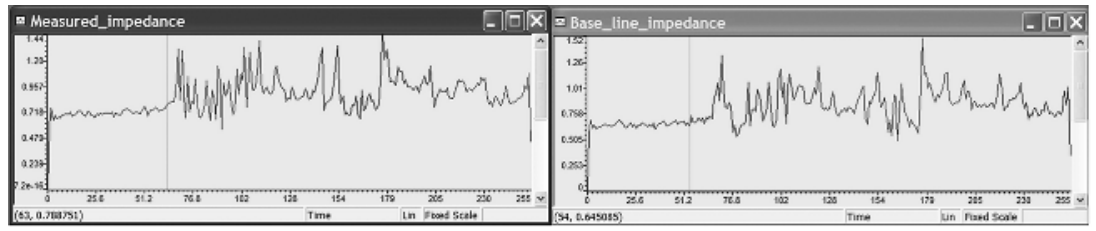

Figure 1.10 The impedance signature for slightly loosening one bolt is compared to the baseline

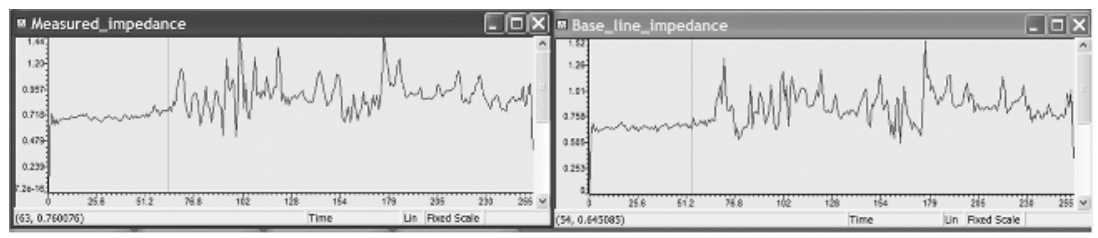

Figure 1.11 The impedance signature for slightly loosening two bolts is compared to the baseline

prototype has successfully detected varying amounts of damage. It is even more promising is that the results are very comparable to an analysis performed with standard impedance measuring equipment, a HP 4194A impedance analyzer.

As another method of validating the prototype device, frequencies displayed by both the HP 4194A impedance analyzer (Figure 1.6) and the new prototype (Figure 1.9) are directly compared. Using the impedance analyzer, data was taken in the range of $10000-32100 \mathrm{~Hz}$. Table 1.3 shows a comparison between selected peaks shown in both Figures 1.6 and 1.9.

Table 1.3 A frequency-by-frequency comparison of the prototype

\begin{tabular}{lccc}
\hline HP 4194A $(\mathrm{Hz})$ & Prototype $(\mathrm{Hz})$ & Difference $(\mathrm{Hz})$ & Difference $(\%)$ \\
\hline 10663 & 10532.9 & 130.1 & 1.22 \\
13094 & 13040.8 & 53.2 & 0.41 \\
15580 & 15548.6 & 31.4 & 0.20 \\
18011.25 & 17931 & 80.25 & 0.45 \\
21215.75 & 21191.2 & 24.55 & 0.12 \\
21547.25 & 21442 & 105.25 & 0.49 \\
26575 & 26457.7 & 117.3 & 0.44 \\
28840.25 & 28714.7 & 125.55 & 0.44 \\
31897 & 31849.5 & 47.5 & 0.15 \\
\hline
\end{tabular}


Obviously, this is only a small sampling of the frequency peaks between 10 and $32 \mathrm{kHz}$. Also, as is expected, each device is slightly more sensitive to some peaks than the other, so some peaks may be missed simply as a function of the frequency resolution. However, even when some peaks may appear to be well apart from one another, they are generally well within the frequency resolution of the machines being used. The impedance analyzer takes data from 10 to $32.1 \mathrm{kHz}$ in 400 points, yielding a frequency resolution of $55.25 \mathrm{~Hz}$. The prototype has a frequency resolution of $125.39 \mathrm{~Hz}$. The percent difference is taken with respect to the HP 4194A impedance analyzer, which is assumed to be the true value for these experiments.

\subsection{HARVESTING, STORAGE AND POWER MANAGEMENT}

In order for autonomous and wireless operation to function, adequate power must be available. Batteries provide sufficient energy to run most wireless transmission systems and computational devices. However, replacing batteries greatly reduces the level of autonomy and limits sensor placement. Hence we propose to develop a system that will recharge itself using ambient vibration and thermal gradients. Some preliminary harvesting results are presented that illustrate the feasibility of recharging batteries from ambient energy.

There are many previous efforts in energy harvesting, especially using the piezoelectric effect. A summary is presented by Sondano et al. (2004b). The book by Roundy et al. (2004) provides a nice introduction to energy harvesting using vibration energy at resonance. The approach by Write et al. is to use resonance as opposed to random excitation used by Sodano et al. Other notable efforts are by Roundy (2005), who again focuses on using resonance to magnify the amount of captured energy, and Guyomar et al. (2005), who use nonlinear circuits to enhance the amount of energy captured. Here we take the approach that random vibration energy is available and that using resonance is not practical.

Depending on the thermal gradients in a given application, harvesting energy from surrounding temperature gradients can provide an order of magnitude more energy than vibration-based harvesting. Thermal energy harvesting represents an important and potentially large source of energy. When propulsion and electronics are operating, heat is generated. Some of this 'waste' energy can be harvested using thermoelectric means, simultaneously keeping these components cool. Also, daily heating and cooling 
provides substantial temperature gradients, which can be exploited. The power generated by thermal gradients can be approximated by

$$
P_{\text {thermal }} \approx \eta A\left(T_{\text {source }}-T_{\text {reject }}\right)
$$

In addition, thermal gradients exist near buildings and machinery (Sodano et al., 2004a,b). Until recently, most solid-state methods relied on large temperature differences to increase the efficiency of the conversion process. Low-energy phenomena are abundant in many waste heat environments around machines and buildings. Thermoelectric coolers (TECs) are designed and manufactured to be most efficient at low temperatures $\left(200^{\circ} \mathrm{C}\right)$, so these devices are excellent thermoelectric generators for low-energy sources. Initial experiments show that power harvested from the ambient vibration and thermal gradients surrounding an internal combustion engine (such as a generator in a village) vary from 18 (vibration) to $70 \mathrm{~mW}$ (thermal).

\subsubsection{Thermal Electric Harvesting}

First consider the problem of capturing ambient waste heat that would be available from aircraft engines, boilers or furnaces. Thermoelectric generators (TEGs) use the Seebeck effect, which describes the current generated when the junction of two dissimilar metals/semiconductors experiences a temperature difference. Using this idea, numerous p-type and n-type junctions are arranged electrically in series, and thermally in parallel, to construct the TEG. Thus, if a thermal gradient is applied to the device, it will generate an electric current that can be utilized to power other electronics. By implementing power harvesting devices, autonomous portable systems can be developed that do not depend on traditional methods for providing power, such as the battery with its limited operating life. The idea to use thermoelectric devices to capture ambient energy from a system is not new. However, TEGs have typically been used simply to determine the extent of power capable of being generated rather than investigating applications and uses of the energy. Furthermore, the majority of previous research efforts have utilized liquid heat exchangers or forced convection to significantly improve heat flow and power generation, but require complex cooling loops and systems. These previous studies commonly do not consider the amount of energy applied to the cooling system and therefore only report gross levels of power. In the present study, thermoelectric generators will be used as power harvesting devices that do not have an active heat exchanger but function as a completely passive energy scavenging system. The motivation for investigating a passive power generation device stems from the need to identify effective power 
sources for the development of self-powered wireless SHM systems. These systems could be placed in a desired location without regular replacement of batteries or maintenance as most wireless devices currently require.

To simulate the energy available in these locations, a hot plate was used. A heat sink was attached to the hot plate to allow more energy to be removed from the cold side of the TEG and facilitate a larger power output. The purpose of this study is to investigate completely passive power harvesting methods, so the systems did not include a means of providing forced convection. The energy produced by the TEG would be greatly increased by forced convection, but active cooling would require additional energy.

The second source of ambient thermal energy tested was waste heat from engines, boilers, lights, etc. To simulate this environment, the TEGs were fixed between a heat sink and a thin aluminum plate, which was then attached to a hot plate using thermal grease. The experimental setup is shown in Figure 1.12. In order to monitor the temperature of the hot and cold side of the TEG, Omega CO-1 thermocouples were used. The thermocouple was only $0.13 \mathrm{~mm}$ thick so it could be placed bonded to the hot and cold sides of the TEG.

A simple circuit has been constructed to take the electrical energy generated by the two energy harvesting devices and store it in a nickel metal hydride battery. The circuit used in this study is shown in Figure 1.13. The diode is a necessary part of this circuit because it forces current to flow only in one direction. If the diode were not present, the TEG would draw power form the battery during times when the voltage generated was less than the voltage of the battery, or, if the hot and cold sides of the TEG switch, a negative voltage will be applied to the battery, causing it to discharge. The output of the TEG is a DC signal, so it does not require a means of rectifying, which is a source of energy loss in piezoelectric power harvesting.

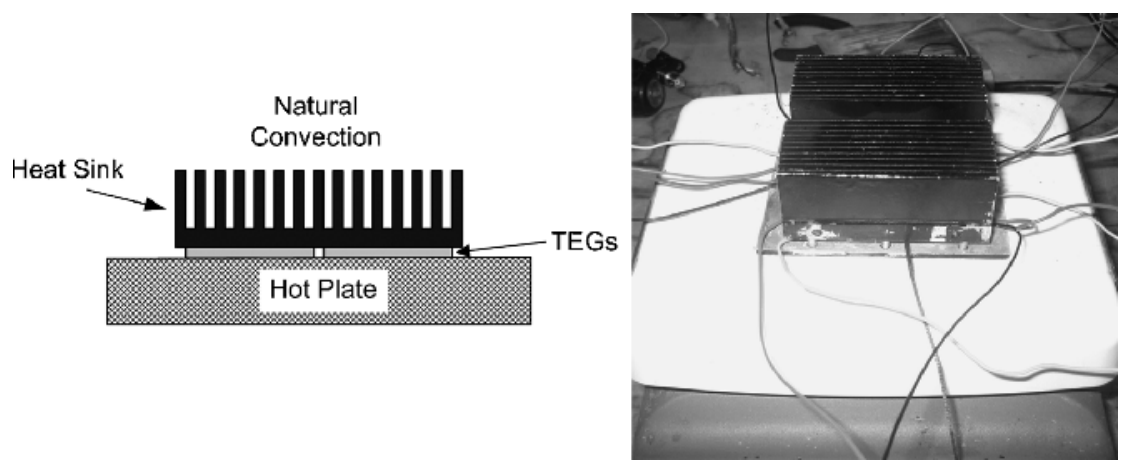

Figure 1.12 Experimental setup of the energy harvesting system 


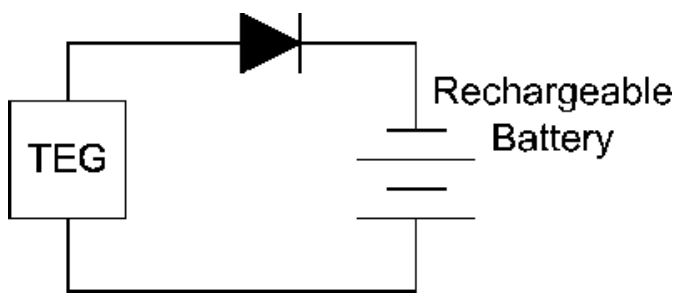

Figure 1.13 Diagram of circuit used to recharge batteries

Table 1.4 Charge times of TEG with solar energy and waste heat, and for a piezoelectric material experiencing random vibration

\begin{tabular}{lccc}
\hline Battery size & $\begin{array}{c}\text { Charge time from } \\
\text { solar energy }\end{array}$ & $\begin{array}{c}\text { Charge time from } \\
\text { waste heat }\end{array}$ & $\begin{array}{c}\text { Charge time from } \\
\text { vibration using } \\
\text { piezoelectric materials }\end{array}$ \\
\hline $80 \mathrm{mAh}$ & $3.3 \mathrm{~min}$ & NA & $2 \mathrm{~h}$ \\
$300 \mathrm{mAh}$ & $17.3 \mathrm{~min}$ & $3.5 \mathrm{~min}$ & $5.8 \mathrm{~h}$ \\
\hline
\end{tabular}

After identifying the ability to use a TEG for the purpose of recharging small batteries, the results were compared to those found using piezoelectric materials by Sodano et al. (2007). The time required for each system to charge the battery to a cell voltage of $1.2 \mathrm{~V}$ was measured in each case and the results are provided in Table 1.4. The results from charging a battery using piezoelectric materials are only provided to give an idea of the results from previous studies performed under realistic conditions and do not mean that a TEG will always outperform piezoelectric materials. The charge times in Table 1.3 may seem to be lower than possible; the time listed is simply to achieve the batteries' cell voltage and not a full charge. The time needed to take the battery up to capacity would be longer; however, the times listed for both the piezoelectric and the TEGs represent the time required to reach the cell voltage from a fully discharged state. In order to determine the time needed to provide a complete charge of the battery, a charge controller would be needed. The superior performance of the TEG is due to its large current output, whereas the piezoelectric material supplies a very high voltage at a low current. To give an idea of the difference in these devices, the impedance of one TEG is approximately $3 \mathrm{ohms}$ while the piezoelectric impedance is approximately $10000 \mathrm{ohms}$. Due to the lower TEG impedance, eight modules had to be connected electrically in series to boost the output voltage to the required $1.2 \mathrm{~V}$ of the battery; however, this lower impedance also 
Table 1.5 Time required to charge different-sized batteries using a piezoelectric

\begin{tabular}{lcc}
\hline Battery size (mAh) & $\begin{array}{c}\text { Time for charge at } \\
\text { resonance (h) }\end{array}$ & $\begin{array}{r}\text { Time for charge with } \\
\text { random signal (h) }\end{array}$ \\
\hline 40 & 0.62 & 1.6 \\
80 & 1.2 & 2 \\
200 & 4 & 1.2 \\
300 & 6 & 5.8 \\
750 & 7 & 8.6 \\
1000 & 22 & 32 \\
\hline
\end{tabular}

makes the TEG far more suited for use with rechargeable batteries, which charge faster with larger currents.

Next, we consider some basic results in harvesting ambient vibration energy from random background vibration such as found near machinery. These are shown in Table 1.5 taken from Sodano et al. (2005a).

\subsubsection{Vibration Harvesting with Piezoceramics}

The piezoelectric effect exists in two domains: the first is the direct piezoelectric effect that describes the material's ability to transform mechanical strain into electrical charge, and the second form is the converse effect, which is the ability to convert an applied electrical potential into mechanical strain energy. The direct piezoelectric effect is responsible for the material's ability to function as a sensor and the converse piezoelectric effect is accountable for its ability to function as an actuator. It is the sensor, or direct, effect that allows piezoelectric material to be used in energy harvesting. A piezoceramic material that is strained, through vibration for example, produces an electric charge which can be bled off and used to produce a voltage and current. Thus piezoceramic materials provide a mechanism to harvest mechanical energy, change it to electrical energy and use it for something else.

Piezoelectric materials belong to a larger class of materials called ferroelectrics. One of the defining traits of a ferroelectric material is that the molecular structure is oriented such that the material exhibits a local charge separation, know as an electric dipole. Throughout the material composition, the electric dipoles are orientated randomly, but when the material is heated above a certain point, the Curie temperature, and a very strong electric field is applied, the electric dipoles reorient themselves relative to the electric field. This process is termed poling. Once the material is cooled, the dipoles 
maintain their orientation and the material is then said to be poled. After the poling process is completed, the material will exhibit the piezoelectric effect.

After the material has been poled, an electric field can be applied in order to induce an expansion or contraction of the material. However, the electric field can be applied along any surface of the material, each resulting in a potentially different stress and strain generation. Therefore, the piezoelectric properties must contain a sign convention to facilitate this ability to apply electrical potential in three directions. For the sake of keeping this discussion simple, the piezoelectric material can be generalized for two cases. The first is the stack configuration that operates in the ' 33 ' mode and the second is the bender, which operates in the ' 13 ' mode. The sign convention assumes that the poling direction is always in the ' 3 ' direction. With this point the two modes of operation can be understood. In the ' 33 ' mode, the electric field is applied in the ' 3 ' direction and the material is strained in the poling or ' 3 ' direction; in the ' 13 ' mode, the electric field is applied in the ' 3 ' direction and the material is strained in the ' 1 ' direction or perpendicular to the poling direction. These two modes of operation are particularly important when defining the electromechanical coupling coefficient that occurs in two forms. The first form is the actuation coefficient $d$, and the second is the sensor coefficient $g$. Thus, $g_{13}$ refers to the sensing coefficient for a bending element poled in the ' 1 ' direction and strained along ' 3 '.

Here we examine some simple lab-based energy harvesting experiments to learn a little about the nature of harvesting using piezoelectric devices. These tests compare the use of monolithic piezoceramics, operating in the $g_{13}$ mode, and active fiber composites, operating in the $g_{33}$ mode. The expectation was that the lighter composites would produce more useable energy than the monolithic piezoceramics because of their higher coupling coefficient. However, this was not the case.

Active fiber composites are layered devices essentially consisting of piezoceramic fibers encased in Kapton and covered with a grid of electrodes (called interdigitated), which results it the $g_{33}$ mode of the piezoceramic being activated when strained. There are two commercially available devices made this way: active fiber composites (AFCs) and macro fiber composites (MFCs), each manufactured in different ways. Here we compare the use of MFCs to monolithic piezoceramics (PZT) in terms of their ability to harvest vibration energy. The experiment consisted of mounting similar-sized MFC and PZT in a cantilever position off of a shaker, exciting the shaker with a random signal measured from a compressor, and running the output of the piezoelectric devices through a bridge circuit for battery charging. 
First, an efficiency of a simple harvesting system is defined. To experimentally determine efficiency a laser vibrometer is used to measure the displacement of a plate covered with a piezoelectric material and a force transducer to measure the applied force. With this data, and the voltage output from the piezoelectric material, the average efficiency $\eta$ was numerically calculated from

$$
\eta=\frac{P_{\text {out }}}{P_{\text {in }}} \times 100 \%=\frac{\sum_{n=2}^{m} \frac{\left(V_{n}-V_{n-1}\right)^{2} / R}{\left(\left(F_{n}-F_{n-1}\right) \cdot\left(d_{n}-d_{n-1}\right)\right) /\left(t_{n}-t_{n-1}\right)}}{m} \times 100 \%
$$

Here $\eta$ is the efficiency, $V$ is the voltage drop across resistance $R, F$ is the force applied to the base of the plate, $d$ is the displacement of the plate, $t$ is the time increment between data points, $n$ is the data point index and $m$ is the highest measured point. The efficiency of three input signals was calculated with the input signals being resonance, chirp and random. The resulting efficiencies are shown in Table 1.6. For each signal three measurements were made to show consistency. The efficiency of the PZT plate is low at resonance because the resonance frequency used was the frequency at which the voltage output was the highest, not the frequency with the best force into voltage out characteristics. This lower efficiency is shown because the resonance frequency is used to charge a battery.

While the MFC had a voltage far larger than the PZT, the power produced was much less. The lower power may be due to the construction of the MFC using piezofibers and interdigitated electrodes providing some additional effects. MFCs are constructed in a diced interdigitated fashion, and the

Table 1.6 Efficiency of PZT and MFC with three different inputs (from Sodano et al., 2003)

\begin{tabular}{lcc}
\hline Signal & PZT efficiency $(\%)$ & MFC efficiency (\%) \\
\hline Resonance & 1.1675 & 0.9442 \\
& 2.0777 & 1.0727 \\
Chirp 0-500 Hz & 1.1796 & 0.8782 \\
& 3.927 & 2.7421 \\
Random 0-500 Hz & 3.9388 & 2.5476 \\
& 3.8948 & 2.6285 \\
& 3.9369 & 0.7636 \\
& 3.6825 & 0.828 \\
& 4.2174 & 0.7366 \\
\hline
\end{tabular}


segments of piezoelectric material between each electrode can be considered a small power source. The majority of these small power sources are connected to one another in series. When two power sources are connected in series the voltages add but the current does not. For this reason, the MFC produces a much higher voltage while the current remains far smaller than that the monolithic PZT. The fiber composite actuators, while promising higher electromechanical coupling than monolithic piezoceramics, are plagued with a low current output, which hinders the rate at which they can charge a battery. This explanation is still under investigation.

The actual results of charging various batteries are given above in Table 1.5. Here the results are only shown for the PZT cases. The important thing to note here is that even with low levels of power, a relatively small amount of random energy can be used to charge up a reasonable-sized battery in a few hours. For the sensing applications we have in mind here, the time required to recharge a battery is well within the useful range for many structures. For instance, if the system of Figure 1.1 is used on a structure that needs to be examined once every $2 \mathrm{~h}$, with the ambient energy required from Table 1.2, then the $200 \mathrm{mAh}$ battery could be used to run the system, take data, compute the damage metric, broadcast the state of health and then put itself to sleep for and hour and a half while the system recharges the battery.

The results here are based on batteries, but one could also use supercapacitors as a storage device. These are compact, lightweight and typically smaller than a battery. The technology is new, but it may just be that supercapacitors will provide an excellent solution to integrate into the system of Figure 1.1.

\subsection{AUTONOMOUS SELF-HEALING}

SHM can be combined with smart materials to form systems capable of healing themselves once damage has been determined. A device-level example is given here, while a materials approach is given in Chapter 9 of this volume. The basic idea is presented here in terms of a bolted joint having the ability to assess its current preload and, if too loose, to tighten itself up (Peairs et al., 2004b; Antonios et al., 2006). The idea is fairly simple: a bolted joint is monitored using, in this case, the impedance-based SHM system. A correlation is made between the impedance profile and the bolt's preload. The bolted joint is fitted with a shape memory alloy (SMA) washer. When the SHM algorithm predicts that the bolt has lost its preload, the SMA washer is activated, causing it to expand and regain the appropriate preload. A schematic of the concept is given in Figure 1.14. 


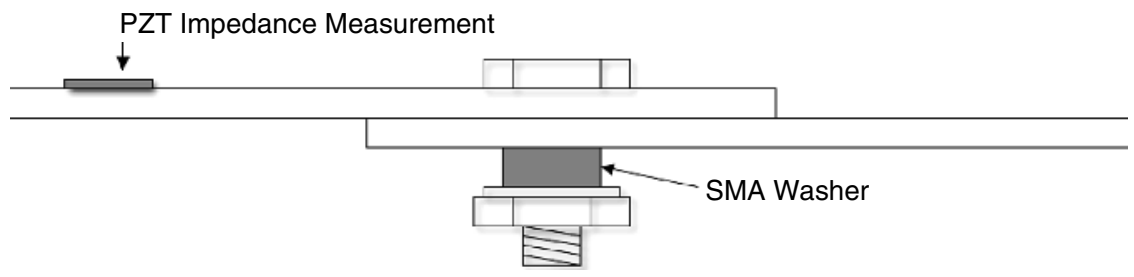

Figure 1.14 A schematic of the self-healing bolted joint concept

SMAs have several distinct features. The shape memory effect is the ability of certain metal alloys to deform as the crystal structure changes from one state (austenitic) at high temperature to another state (martensitic) at low temperature. This phase transition is due to twinning and de-twinning crystal planes. The material deformation occurs as a result of the movement of twin planes without moving dislocations. This allows strains to be easily recovered, and the material appears to remember its shape. Up to $8 \%$ strain can be recovered in this way. Here the SMA is used in constrained recovery where heat is applied to cause it to expand to its original configuration.

Initial experiments were done to show the ability of SMA washers to restore preload in loosened joints. A $0.965 \mathrm{~cm}$ thick cylindrical SMA (Nitinol) washer was located between the nut and clamped members, as seen in Figure 1.14. The washer had an inner diameter of $2.436 \mathrm{~cm}$ and outer diameter of $2.677 \mathrm{~cm}$.

The impedance response function was measured with the bolt tightened to $40.7 \mathrm{Nm}$, representing its undamaged state. The bolt was then loosened to $13.6 \mathrm{~N} \mathrm{~m}$ to represent damage, and the impedance response function was again measured. Note that while this seems quite a lot, the bolt is still tight, and this amounts to about a quarter turn of the bolt. The entire structure was then heated in an oven to actuate the SMA. The actuator expanded axially (as well as contracting radially) to restore the preload in the joint. A final impedance measurement was then taken. The response of the beam after actuation returns from the damaged state to a state near that of the undamaged condition. After actuation and removal from the joint, the actuator was $0.979 \mathrm{~cm}$ thick and had an inner diameter of $2.349 \mathrm{~cm}$ and outer diameter of $2.624 \mathrm{~cm}$. These simple experiments motivated sorting out a device to automate the procedure and provide local heating.

SMA can be heated by passing a current through it and inducing resistive heating. Unfortunately, this also tends to heat up all the other metal in the lap joint, including the beams, requiring significant amounts of energy. The solution is to provide a local heater to wrap around the SMA washer and 
thermally insulate it. With the temperature controlled by a heater, one is able to formulate a model to predict the preload as a function of temperature. This model in turn can help map the impedance measurements to the value of temperature that needs to be applied in order to return the system to its designed preload. This is reported in Antonios et al. (2006).

Once this system is designed, the decision to 'fire' the SMA washer can be made in an automated way by continually monitoring plots such as those of Figure 1.7. Once the impedance metric exceeds a predetermined value, a decision circuit can turn on the heater to a specified temperature, regaining the desired preload. This concept illustrates a 'Level 7' system as described in Section 1.2 and forms an example of a 'self-healing system'. This system of Figure 1.14 is self-healing in the sense that it: determines that damage exists; determines how severe the damage is; and then takes action to recover from the damage and restore the system's original function.

\subsection{THE WAY FORWARD: AUTONOMIC STRUCTURAL SYSTEMS FOR THREAT MITIGATION}

Military and security issues often drive research. In this case, one of the motivators for having autonomous sensing is the desire of several governments to mitigate threats against their military and civilians. The US military's research establishments (Air Force Office of Scientific Research, Army Research Office, and Office of Naval Research) combined with the US National Science Foundation and the European Science Foundation held a workshop in May of 2006 on 'Autonomic Structural Systems for Threat Mitigation'. Autonomic structural systems are loosely defined as systems which respond to external threat in an autonomous way. The workshop's focus was on load-bearing composite material structures with the hope of producing a road map forward for developing multifunctional materials and structures capable of (a) sensing and diagnosing of threats, (b) penetration prevention, (c) load capacity preservation and (d) functionality restoration.

Developing examples of autonomic systems will require the collaboration of engineers and scientists from a variety of disciplines and should be motivated or inspired by looking at biological solutions to threat mitigation (such as bone regrowth). Certain components of the proposed autonomic systems already exist. There are several contributors to this volume who have looked at self-healing, some who have looked at autonomous sensing (as in this chapter and the references) and some who have looked at load-bearing 
sensors. The concept of multifunctional structures has been around for some time. The import of the proposed autonomic structural and material systems is to bring yet another level of integration together with the hopes of producing structures that autonomously 'take care of themselves' under a variety of threats.

Primitive elements of autonomic systems already exist in the form of airbags and crush zones in automobiles, the wireless sensing and power harvesting systems mentioned above, and the recent work in self-healing materials and structures. The way forward proposes a more sophisticated level of integration and autonomy. Components of embedded neural computing and multifunctional sensing are proposed, with innovations demanded in three areas:

1. Multifunctional sensing and actuation

2. Integrated sensing, computing, informatics and communication

3. Predictive and proactive sensing

Multifunctional sensing refers to the concept of a mobile, multidirectional sensor that could respond to a variety of length and time scales, change its sensing mechanism (say from acoustic pressure waves to chemical), change its orientation and reprogram its function. Actuation refers to mechanical, chemical and electromagnetic force. Actuation and sensing can be combined to form the concept of a morphing sensor that could change its physical form to adjust to the appropriate threat. These ideas are inspired by the many living creatures on Earth and the way in which our own bodies respond to threats and/or damage.

While these ideas may seem far fetched by today's standards, they motivate the scientific and technological issues of (a) expanding the 'abilities' of our current sensing systems and materials, (b) integrating sensor functions, actuation and intelligence (i.e. computing), (c) expanding current functionality and levels of integration, and (d) improving our energy harvesting and power management systems.

Our own particular efforts in these directions focus on continuing to integrate multiple energy harvesting methods into a single load-bearing structure. We are further pursuing chip computing without an operating system to minimize the amount of power needed to run algorithms at the sensor location (integrated computing). On the harvesting front, we hope to examine a particular application of a micro air vehicle to extend its range and performance by integrating multiple harvesting sources into structural components and focusing on video sensing. In addition, we hope to miniaturize our current 
prototype described above to provide a more highly integrated structural sensor that is completely autonomous. While these are minor perturbations of our existing systems in the context of the way forward to an autonomous system, they form necessary steps to eventually achieving an autonomic structure.

\subsection{SUMMARY}

This chapter presents the first fully self-contained system that performs impedance-based SHM. In previous research, a system was developed which performed most of the health monitoring steps, but needed the use of an external function generator for actuation. The current autonomous system effectively replaces an impedance analyzer and external data analysis. All of the structural excitation, data acquisition and health monitoring analysis are performed in a matter of seconds. With traditional impedance techniques, after the data is acquired, all of the analysis must still be done using processing software to determine whether there is damage. Now, damage in a structure can be found almost immediately.

Also described is the first use of impedance excitation with targeted sinc functions. The use of sinc functions has the potential to save both excitation time and computational power. By slightly varying the fundamental frequency with each pulse, the structure will be excited at every frequency in the range of interest.

A quick review of methods for capturing ambient vibration and thermal energy for use in for battery charging is also presented. The idea proposed here is to produce a completely wireless system capable of providing autonomous structural health monitoring.

A simple example of a self-healing bolted joint is also presented as an illustration of an autonomous self-monitoring and healing system. All of this leads to the future direction of adaptive, autonomous structures as described above. In the short term, future work on the autonomous system includes performing a complete excitation signal energy analysis to explore the benefits of actuation with sinc functions. Also, piezoelectricbased and thermal power harvesting will be incorporated to allow the system to be fully self-sufficient. Eventually, with the knowledge gained from this prototype, an even smaller prototype can be custom designed with components specific to the project, all leading to the eventual goal of having a complete impedance-based SHM system contained on a single chip. 


\section{ACKNOWLEDGEMENTS}

The authors would especially like to thank Hyung-Jin Lee, advised by Dr. Dong S. Ha, of the Virginia Tech Electrical and Computer Engineering Department, for all his hard work and help on this project. Dr. Robert Owen of Extreme Diagnostics, Inc. and Dr. Gyuhae Park of Los Alamos National Laboratory also contributed to this research. The authors gratefully acknowledge funding for this research provided by NASA Langley Research Center and Extreme Diagnostics, Inc. (contract NNL05AB08P). This material is based in part upon work supported by the National Science Foundation under Grant No. 0426777.

\section{REFERENCES}

Antonios, C., Inman, D.J. and Smaili, A., 2006, 'Experimental and Theoretical Behavior of Self-healing Bolted Joints,' Journal of Intelligent Material Systems and Structures, 17(6), pp. 499-509.

Doebling, S.W., Farrar, C.R. and Prime, M.B., 1998, 'A Summary Review of Vibration-Based Damage Identification Methods,' Shock and Vibration Digest, 30(2), pp. 91-105.

Dosch, J.J., Inman, D.J. and Mayne, R.W., 1994, 'Dual Function System Having a Piezoelectric Element (Self Sensing Actuator),' US Patent \#5,347,870 awarded September 20, 1994.

Giurgiutiu, V. and Zagrai, A.N., 2002, 'Embedded Self-Sensing Piezoelectric Active Sensors for On-line Structural Identification,' Journal of Vibration and Acoustics, 124, pp. 116-125.

Grisso, B.L., Martin, L.A. and Inman, D.J., 2005, 'A Wireless Active Sensing System for Impedance based Structural Health Monitoring', Proceedings of IMAC XXIII, Orlando, FL, January 31-February 3.

Guyamor, D., Badel, D., Leveuvre, E. and Richard, C., 2005, 'Toward Energy Harvesting using Active Materials and Conversion Improvement by Nonlinear Processing', IEEE Transactions on Ultrasonics, Ferroelectrics and Frequency Control, 53(4), pp. 584-596.

Inman, D.J., Farrar, C.R., Steffan, V. and Lopez, V., Ed., 2005, Damage Prognosis, John Wiley and Sons, Ltd, Chichester.

Liang, C., Sun, F.P. and Rogers, C.A., 1994, 'An Impedance Method for Dynamic Analysis of Active Material System,' Journal of Vibration and Acoustics, 116, 121-128.

Lynch, J.P. and Loh, K., 2005, 'A Summary Review of Wireless Sensors and Sensor Networks for Structural Health Monitoring,' Shock and Vibration Digest, 38(2), pp. 91-128.

Lynch, J.P., Sundararajan, A., Law, K.H., Kiremidjian, A.S., Carryer, E., Sohn, H. and Farrar, C.R., 2003, 'Field Validation of a Wireless Structural Monitoring System on the Alamosa Canyon Bridge,' SPIE's 10th Annual International Symposium on Smart Structures and Materials, San Diego, CA. 
Lynch, J.P., Sundararajan, A., Law, K.H., Kiremidjian, A.S., Kenny, T.W. and Carryer, E., 2002, 'Embedment of Structural Monitoring Algorithms in a Wireless Sensing Unit,' Structural Engineering and Mechanics, 15(3), pp. 285-297.

Lynch, J.P., Sundararajan, A., Law, K.H., Kiremidjian, A.S. and Carryer, E., 2004a, 'Embedding Damage Detection Algorithms in a Wireless Sensing Unit for Attainment of Operational Power Efficiency.' Smart Materials and Structures, 13, pp. 800-810.

Lynch, J.P., Sundararajan, A., Law, K.H., Sohn, H. and Farrar, C.R., 2004b, 'Piezoelectric Structural Excitation Using a Wireless Active Sensing Unit', Proceedings of the 22nd International Modal Analysis Conference, on CD by the Society of Experimental Mechanics.

Mascarenas, D.L., Todd, M.D., Park, G. and Farrar, C.R., 2006, 'A Miniaturized Electromechanical Impedance-based Node for the Wireless Interrogation of Structural Health', Proceeding of SPIE's 13th Annual International Symposium on Smart Structures and Materials, 6177, March 28.

Park, G., Sohn, H., Farrar, C.R. and Inman, D.J., 2003, 'Overview of Piezoelectric Impedance-Based Health Monitoring and Path Forward,' Shock and Vibration Digest, 35(6), 451-463.

Peairs, D.M., Park, G. and Inman D.J., 2004a. 'Improving Accessibility of the Impedance-based Structural Health Monitoring Method,' Journal of Intelligent Material Systems and Structures, 15(2), 129-140.

Peairs, D.M., Park, G. and Inman, D.J., 2004b, 'Practical Issues of Activating Selfrepairing Bolted Joints,' Smart Materials and Structures, 13, pp. 1414-1423.

Radiometrix Ltd., 2005, UHF Narrow Band FM multi channel radio modules, Radiometrix Inc., Harrow, Middlesex.

Roundy, S., 2005, 'On the Effectiveness of Vibration-Based Energy Harvesting,' Journal of Intelligent Material Systems and Structures, 16, pp. 809-823.

Roundy, S., Wright, P.K. and Rabaey, J.M. 2004, Energy Scavenging for Wireless Sensor Networks: With Special Focus on Vibrations, Kluwer Academic, Dordrecht.

Schilling, R.J. and Harris, S.L., 2005, Fundamentals of Digital Signal Processing using MATLAB, Nelson, Toronto.

Sodano, H.A., Park, G., Leo, D.J. and Inman, D.J., 2003, 'Use of Piezoelectric Energy Harvesting Devices for Charging Batteries,' Proceeding of SPIE's 10th Annual International Symposium on Smart Structures and Materials, 5050, pp. 101-108, July.

Sodano, H.A, Park, G. and Inman, D.J., 2004a, 'Estimation of Electric Charge Output for Piezoelectric Energy Harvesting,' Journal of Strain, 40, pp. 49-58.

Sodano, H., Park, G. and Inman, D.J., 2004b. 'A Review of Power Harvesting from Vibration using Piezoelectric Materials,' Shock and Vibration Digest, 36(3), pp. 197-205.

Sodano, H.A., Park, G. and Inman, D.J., 2005a, 'Comparison of Piezoelectric Energy Harvesting Devices for Recharging Batteries,' Journal of Intelligent Material Systems and Structures, 16(10), pp. 799-808.

Sodano, H.A., Inman, D.J. and Park, G., 2005b. 'Generation and Storage of Electricity from Power Harvesting Devices,' Journal of Intelligent Material Systems and Structures, 16(1), pp. 67-75. 
Sodano, H.A., Simmers, G.E., Dereux, R. and Inman, D.J., 2007, 'Recharging Batteries Using Energy Harvested from Thermal Gradients,' Journal of Intelligent Material Systems and Structures, 18(1), pp. 3-10.

Sohn, H., Allen, D.W., Worden, K. and Farrar, C.R., 2003, 'Statistical Damage Classification Using Sequential Probability Ratio Tests,' An International Journal of Structural Health Monitoring, 2, pp. 57-74.

Sohn, H., Worden, K. and Farrar, C.R., 2002, 'Statistical Damage Classification Under Changing Environmental and Operational Conditions,' Journal of Intelligent Material Systems and Structures, 13, pp. 561-574.

Spencer, Jr., B.F., Ruiz-Sandoval, M.E. and Kurata, N., 2004, 'Smart Sensing Technology: Opportunities and Challenges,' Journal of Structural Control and Health Monitoring, 11(4), 349-368.

Straser, E.G. and Kiremidjian, A.S., 1998, A modular, wireless damage monitoring system for structures. Report No. 128, John A. Blume Earthquake Engineering Center, Department of Civil and Environmental Engineering, Stanford University, Stanford, CA.

Tanner, N.A, Wait, J.R., Farrar, C.R. and Sohn, H., 2003, 'Structural Health Monitoring Using Wireless Sensing Systems with Embedded Processing', Journal of Intelligent Materials Systems and Structures, 14(1), pp. 43-55.

Texas Instruments, 2005a, Code Composer Studio Development Tools v3.1 Getting Started Guide, Texas Instruments Inc., Dallas, Texas.

Texas Instruments, 2005b, TMS320C6713, TMS320C6713B Floating-Point Digital Signal Processors, Texas Instruments Inc., Dallas, Texas.

Texas Instruments, 2002, ADS8364EVM User's Guide, Texas Instruments Inc., Dallas, Texas.

Texas Instruments, 2001, TLV5619-5639 12-Bit Parallel DAC Evaluation Module User's Guide, Texas Instruments Inc., Dallas, Texas. 